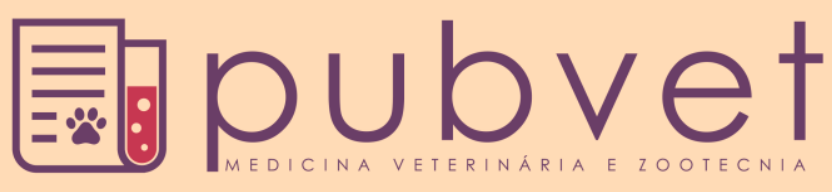

https://doi.org/10.22256/pubvet.v12n5a98.1-6

\title{
Produtividade de forragem e composição química de Stylosanthes capitata cv. Lavradeiro sob níveis de fósforo
}

\author{
Newton de Lucena $\operatorname{Costa}^{1^{*}}$, Valdinei Tadeu Paulino ${ }^{2} \bullet$, Vicente Gianluppi ${ }^{3} \bullet$, Amaury $^{\circ}$ \\ Burlamaqui Bendahan $^{1 \oplus}$, João Avelar Magalhães ${ }^{4} \bullet$
}

${ }^{1}$ Eng. Agr., D.Sc., Pesquisador da Embrapa Roraima, Boa Vista, RR.

${ }^{2}$ Eng. Agr., Ph.D., Pesquisador do Instituto de Zootecnia, Nova Odessa, SP.

${ }^{3}$ Eng. Agr.,M.Sc., Pesquisador da Embrapa Roraima, Boa Vista, RR.

${ }^{4}$ Med. Veterinário, D.Sc., Pesquisador da Embrapa Meio-Norte, Parnaíba, PI.

*Autor para correspondência, E-mail: newton.lucena-costa@embrapa.br

\begin{abstract}
RESUMO. O efeito de níveis de fósforo (0, 40, 80 e $120 \mathrm{~kg}$ de $\left.\mathrm{P}_{2} \mathrm{O}_{5} \mathrm{ha}^{-1}\right)$ sobre o rendimento de matéria seca verde (MSV), composição química e nodulação de Stylosanthes capitata cv. Lavradeiro, foi avaliado sob condições de campo no cerrado de Roraima. A adubação fosfatada afetou significativamente $(\mathrm{P}<0,05)$ os rendimentos de MSV, teores de nitrogênio $(\mathrm{N})$, fósforo $(\mathrm{P})$, cálcio $(\mathrm{Ca})$, magnésio $(\mathrm{Mg})$ e potássio $(\mathrm{K})$, número e peso seco de nódulos. O máximo rendimento de MSV e os maiores teores de $\mathrm{Ca}$, $\mathrm{Mg}$ e K foram obtidos com a aplicação de 89,4; 56,2; 113,1 e 75,6 kg de $\mathrm{P}_{2} \mathrm{O}_{5} \mathrm{ha}^{-1}$, respectivamente, enquanto que os teores de $\mathrm{N}, \mathrm{P}$ e o número de nódulos foram diretamente proporcionais aos níveis de $\mathrm{P}$. O nível crítico interno de $\mathrm{P}$, relacionado com $90 \%$ do rendimento máximo de MSV, foi estimado em $2,43 \mathrm{~g} \mathrm{~kg}^{-1}$. A eficiência de utilização de $\mathrm{P}$ foi inversamente proporcional ás doses aplicadas.
\end{abstract}

Palavras-chave: composição química, matéria seca verde, nodulação

\section{Forage production and chemical composition of Stylosanthes capitata cv. Lavradeiro under phosphorus levels}

\begin{abstract}
The effects of phosphorus levels $\left(0,40,80\right.$ and $120 \mathrm{~kg}$ of $\left.\mathrm{P}_{2} \mathrm{O}_{5} \mathrm{ha}^{-1}\right)$ on green dry matter (GDM), chemical composition and nodulation of Stylosanthes capitata cv. Lavradeiro were evaluated under field conditions in Roraima's savannas. Phosphorus fertilization increased significantly $(\mathrm{P}<.05)$ GDM yields, nitrogen $(\mathrm{N})$, phosphorus $(\mathrm{P})$, calcium $(\mathrm{Ca})$, magnesium $(\mathrm{Mg})$ and potassium $(\mathrm{K})$ concentrations and number and dry weight of nodules. Maximum GDM yields and $\mathrm{Ca}, \mathrm{Mg}$ and $\mathrm{K}$ contents were obtained with the application of $89.4 ; 56.2 ; 113.1$ and $75.6 \mathrm{~kg}_{\text {o }} \mathrm{P}_{2} \mathrm{O}_{5} \mathrm{ha}^{-1}$, respectively, while the $\mathrm{N}$ and $\mathrm{P}$ contents and number of nodules were directly proportional to $\mathrm{P}$ levels. The $\mathrm{P}$ critic level, related to $90 \%$ of GDM maximum production, was estimated at $2.43 \mathrm{~g} \mathrm{~kg}^{-1}$. The $\mathrm{P}$ efficiency utilization was inversely proportional to the $\mathrm{P}$ rates applied.
\end{abstract}

Keywords: chemical composition, green dry matter, nodulation

\section{Productividad de forraje y composición química de Stylosanthes capitata cv. Lavradeiro bajo niveles de fósforo}

RESUMEN. El efecto de niveles de fósforo $\left(0,40,80\right.$ y $120 \mathrm{~kg}$ de $\left.\mathrm{P}_{2} \mathrm{O}_{5} \mathrm{ha}^{-1}\right)$ sobre el rendimiento de materia seca verde (MSV), composición química y nodulación de Stylosanthes capitata cv. Lavradeiro, fue evaluado bajo condiciones de campo en las sabanas de Roraima. La fertilización fosfatada incrementó significativamente $(\mathrm{P}<0,05)$ 
los rendimientos de MSV, los contenidos de nitrógeno $(\mathrm{N})$, fósforo $(\mathrm{P})$, calcio $(\mathrm{Ca})$, magnesio $(\mathrm{Mg})$ y potasio $(\mathrm{K})$, el número y el peso seco de los nódulos. El máximo rendimiento de MSV y los mayores contenidos de $\mathrm{Ca}, \mathrm{Mg}$ y $\mathrm{K}$ fueron obtenidos con la aplicación de 89,4; 56,2; 113,1 y 75,6 kg de $\mathrm{P}_{2} \mathrm{O}_{5} \mathrm{ha}^{-1}$, mientras que los contenidos de $\mathrm{N}$, $\mathrm{P}$ y el número de nódulos fueron directamente proporcionales a los niveles de $\mathrm{P}$. El nivel crítico interno de $\mathrm{P}$, relacionado con el $90 \%$ del rendimiento máximo de MSV, fue estimado en $2,43 \mathrm{~g} \mathrm{~kg}^{-1}$. La eficiencia de utilización de $\mathrm{P}$ fue inversamente proporcional a las dosis aplicadas.

Palabras clave: composición química, materia seca verde, nodulación

\section{Introdução}

Em Roraima, os solos sob vegetação de cerrados apresentam baixa fertilidade natural, o que reduz o potencial da produtividade biológica primária das pastagens cultivadas, notadamente das leguminosas forrageiras tropicais (Gianluppi et al., 2002). Ademais, a utilização de práticas de manejo inadequadas (elevadas cargas animais, sistema de pastejo contínuo e ausência de fertilizações de estabelecimento e/ou manutenção), as quais afetam consideravelmente a eficiência dos processos de reciclagem de nutrientes, o aparecimento de deficiências de fósforo $(\mathrm{P})$ tem sido frequente e intenso. Ensaios exploratórios de fertilidade do solo realizados na região amazônica demonstraram que $\mathrm{o} P$ foi $\mathrm{o}$ nutriente mais limitante ao crescimento de diversas leguminosas forrageiras (Arachis pintoi, Centrosema pubescens, Pueraria phaseoloides e Stylosanthes guianensis), reduzindo drasticamente seus rendimentos de forragem, número e peso seco de nódulos, teores de nitrogênio e P (Costa et al., 2009).

No estabelecimento, formação e manejo de pastagens cultivadas, o conhecimento dos fatores nutricionais limitantes ao crescimento de leguminosas forrageiras tropicais torna-se imprescindível para a formulação de práticas adequadas de adubação (Costa et al., 2006; Lopes et al., 2011). O P desempenha importante papel no desenvolvimento do sistema radicular, na ramificação e na maximização dos processos de fixação biológica de nitrogênio das leguminosas, sendo indispensável à fotossíntese, síntese e degradação dos carboidratos, além de participar ativamente da respiração celular, influenciando o armazenamento, transporte e utilização da energia produzida no processo fotossintético, o que contribui para maior absorção de água e nutrientes (Fabrice et al., 2015; Moura et al., 2011). Considerando-se o elevado investimento na aquisição de fertilizantes fosfatados e sua importância relativa na composição dos custos de produção dos sistemas pecuários, torna-se necessário assegurar sua máxima eficiência, através da determinação das doses mais adequadas para o estabelecimento e manutenção das pastagens (Carneiro et al., 2011; Costa et al., 2007; Lopes et al., 2011).

Dentre as várias leguminosas forrageiras introduzidas e avaliadas em Roraima, Stylosanthes capitata cv. Lavradeiro destacou-se como uma das mais promissoras, apresentando excelente produtividade de forragem, bom valor nutritivo e grande capacidade de colonização do solo, além de boa adaptação a solos de baixa fertilidade natural, alta tolerância à seca e excelente regeneração natural, em decorrência da produção de grandes quantidades de sementes (Gianluppi et al., 2002; Costa et al., 2009).

Neste trabalho avaliou-se o efeito de níveis de $P$ sobre a produção de forragem, composição química e nodulação de $S$. capitata cv. Lavradeiro nos cerrados de Roraima.

\section{Material e Métodos}

O ensaio foi conduzido no Campo Experimental da Embrapa Roraima, localizado em Boa Vista, durante o período de maio a setembro de 2015, o qual correspondeu a uma precipitação acumulada de $1.218,9 \mathrm{~mm}$ e temperatura média mensal de $24,86^{\circ} \mathrm{C}$. O solo da área experimental é um Latossolo Amarelo, fase cerrado, textura média, com as seguintes características químicas, na profundidade de 0-20 cm: $\mathrm{pH}_{\mathrm{H} 2 \mathrm{O}}=4,9 ; \mathrm{P}=2,5 \mathrm{mg} / \mathrm{kg} ; \mathrm{Ca}+\mathrm{Mg}=1,15$ $\mathrm{cmol}_{\mathrm{c}} \cdot \mathrm{dm}^{-3} ; \mathrm{K}=0,019 \mathrm{cmol}_{\mathrm{c}} \cdot \mathrm{dm}^{-3}$ e $\mathrm{Al}=0,59$ $\mathrm{cmol}_{c} \mathrm{dm}^{-3}$.

O delineamento experimental foi inteiramente ao acaso com três repetições. Os tratamentos consistiram de quatro níveis de adubação fosfatada $\left(0,40,80\right.$ e $120 \mathrm{~kg}$ de $\left.\mathrm{P}_{2} \mathrm{O}_{5} \mathrm{ha}^{-1}\right)$, aplicados sob a forma de superfosfato triplo. As parcelas mediam 2,0 × 2,0 m, sendo a área útil de $1,0 \mathrm{~m}^{2}$. A adubação de estabelecimento constou 
da aplicação de $80 \mathrm{~kg}$ de $\mathrm{K}_{2} \mathrm{O}$ ha $^{-1}$, sob a forma de cloreto de potássio. A aplicação do $\mathrm{P}$ foi parcelada em duas vezes, sendo metade quando do plantio da leguminosa e metade decorridos 35 dias, por ocasião da primeira avaliação da produtividade de forragem. A leguminosa foi semeada a lanço e sem nenhum revolvimento do solo, utilizando-se densidade de semeadura de $3,0 \mathrm{~kg} \mathrm{ha}^{-1} \mathrm{de}$ sementes (Valor cultural $=100 \%$ ). Durante o período experimental foram realizados quatro cortes a intervalos de 35 dias e a $20 \mathrm{~cm}$ acima do solo.

Os parâmetros avaliados foram rendimento de matéria seca verde (MSV), teores de nitrogênio $(\mathrm{N})$, fósforo $(\mathrm{P})$, cálcio $(\mathrm{Ca})$, magnésio $(\mathrm{Mg})$ e potássio (K) e nodulação (número e peso seco de nódulos). Os teores $\mathrm{N}$ foram analisados de acordo com procedimentos descritos por Silva \& Queiroz (1981); enquanto que os teores P, Ca, $\mathrm{Mg}$ e $\mathrm{K}$ foram determinados conforme a metodologia descrita por Silva (2009). Os teores de $\mathrm{P}$ e $\mathrm{K}$ foram quantificados após digestão nitroperclórica. $\mathrm{O} \quad \mathrm{P}$ foi determinado por colorimetria; o K por fotometria de chama e $\mathrm{Ca}$ e $\mathrm{Mg}$ por espectrofotometria de absorção atômica. Para obtenção da eficiência de uso do potássio (EUP) foi utilizada a equação: EUP = MSV total na dose $\mathrm{P}$ - MSV na dose zero $\div$ dose de $\mathrm{P}$ aplicada. O nível crítico interno (NCI) de $\mathrm{P}$ foi determinado ajustando-se a equação de regressão para rendimento de MSV (variável dependente) e níveis de $\mathrm{P}$ (variável independente) (equação 1) e para teores de $\mathrm{P}$ como variável dependente dos níveis de $\mathrm{P}$ aplicados (equação 2). Através da equação 1 calculou-se a dose de $\mathrm{P}$ aplicada relativa a $90 \%$ do rendimento máximo de MSV, sendo este valor substituído na equação 2 . A avaliação da nodulação foi realizada através da coleta de três plantas/parcela. As raízes foram separadas do solo utilizando-se jatos de água corrente. A seguir, procedeu-se a remoção, purificação, contagem e pesagem dos nódulos.

Os dados foram submetidos à análise de variância e de regressão considerando o nível de significância de $5 \%$ de probabilidade. Para se estimar a resposta dos parâmetros avaliados aos níveis de $\mathrm{P}$, a escolha dos modelos de regressão baseou-se na significância dos coeficientes linear e quadrático, por meio do teste " $t$ ", de Student, ao nível de $5 \%$ de probabilidade.

\section{Resultados e Discussão}

Os efeitos da adubação fosfatada sobre os rendimentos de MSV foram ajustados ao modelo quadrático de regressão e o máximo valor estimado com a aplicação de $89,4 \mathrm{~kg}$ de $\mathrm{P}_{2} \mathrm{O}_{5}$ ha $^{-1}$ (Tabela 1). Em pastagens de Stylosanthes guianensis cv. Mineirão, Costa et al. (2007) constataram incrementos na disponibilidade de forragem de $81,3 \%$ (2.857 $\mathrm{kg}$ de MSV ha-1) e $103,7 \%$ (3.210 kg de MSV ha-1) com a aplicação de 50 e $100 \mathrm{~kg}$ de $\mathrm{P}_{2} \mathrm{O}_{5} \mathrm{ha}^{-1}$, respectivamente, comparativamente ao tratamento controle (1.576 $\mathrm{kg}$ de MSV $\mathrm{ha}^{-1}$ ). Os rendimentos de MSV registrados foram superiores aos relatados por Costa et al. (2006) para pastagens de Stylosanthes guianensis cv. Bandeirante fertilizadas com 60, 90 e $120 \mathrm{~kg}$ de $\mathrm{P}_{2} \mathrm{O}_{5}$ ha $^{-1}$ ano $^{-1}(1.423 ; 1.833$ e $2.578 \mathrm{~kg}$ de MSV ha- ${ }^{-1}$, respectivamente).

A EUP foi inversamente proporcional às doses utilizadas e ajustada ao modelo linear de regressão, no entanto a leguminosa apresentou maior responsividade que a relatada por Costa et al. (2007) para pastagens de S. macrocephala cv. Pioneiro $\left(15,2 ; 11,5\right.$ e 5,3 kg de MSV/kg de $\mathrm{P}_{2} \mathrm{O}_{5}$ $\mathrm{ha}^{-1}$, respectivamente para doses de 50,75 e 100 $\mathrm{kg}$ de $\mathrm{P}_{2} \mathrm{O}_{5} \mathrm{ha}^{-1}$ ) (Tabela 1). A EUP reflete o grau de recuperação do nutriente pelas culturas, considerando as perdas por lixiviação, fixação e as que geralmente ocorrem durante os processos de absorção, translocação e utilização (Costa et al., 2012; Otsubo et al., 2011). A adubação fosfatada afetou positivamente a produtividade de MSV, contudo, maiores doses apresentaram menor eficiência de utilização. Simão Neto et al. (1999) para S. guianensis cv. Cook e Costa et al. (2007) para Cajanus cajan reportaram maiores EUP com a aplicação de $40(21,2 \mathrm{~kg}$ de MSV $/ \mathrm{kg}$ de $\left.\mathrm{P}_{2} \mathrm{O}_{5} \mathrm{ha}^{-1}\right)$ e $60 \mathrm{~kg}$ de $\mathrm{P}_{2} \mathrm{O}_{5} \mathrm{ha}^{-1}(26,1 \mathrm{~kg}$ de $\mathrm{MSV} / \mathrm{kg}$ de $\mathrm{P}_{2} \mathrm{O}_{5}$ ha $\left.^{-1}\right)$, respectivamente.

Os teores de $\mathrm{Ca}, \mathrm{Mg}$ e $\mathrm{K}$ da leguminosa foram ajustados ao modelo quadrático de regressão e os maiores valores estimados com a aplicação de 56,$2 ; 113,1$ e $75,6 \quad \mathrm{~kg}$ de $\mathrm{P}_{2} \mathrm{O}_{5} \quad \mathrm{ha}^{-1}$, respectivamente, enquanto que para o $\mathrm{N}$ e o $\mathrm{P}$ os efeitos da adubação fosfatada foram lineares e positivos (Tabela 1). O decréscimo na concentração de nutrientes, com o desenvolvimento do dossel da pastagem, em função do maior acúmulo de forragem decorrente da adubação fosfatada, decorre de dois fatores: 1) decréscimo na razão de área foliar (relação entre área foliar e a biomassa da planta), face ao maior investimento dos nutrientes na produção de biomassa estrutural e que não apresenta função fotossintetizante, visando promover o aumento em altura e tornar as plantas mais competitivas na 
captação da radiação incidente e; 2) decréscimo na concentração de nutrientes por unidade de área foliar das folhas sombreadas na porção inferior da planta, priorizando a alocação de nutrientes nas folhas superiores que apresentam maior taxa de fotossíntese (Lemaire \& Agnusdei, 2000; Lemaire et al., 2011).

Tabela 1. Rendimento de matéria seca verde (MSV - $\mathrm{kg} \mathrm{ha}^{-1}$ ), eficiência de utilização de fósforo (EUP - $\mathrm{kg}$ de MSV/kg de $\left.\mathrm{P}_{2} \mathrm{O}_{5} \mathrm{ha}^{-1}\right)$, teores $\left(\mathrm{g} \mathrm{kg}^{-1}\right)$ de nitrogênio $(\mathrm{N})$, fósforo $(\mathrm{P})$, cálcio $(\mathrm{Ca})$, magnésio $(\mathrm{Mg})$ e potássio $(\mathrm{K})$, número de nódulos $(\mathrm{NN})$ e peso seco de nódulos (mg) (PSN) de Stylosanthes capitata cv. Lavradeiro, em função da adubação fosfatada. Médias de quatro cortes

\begin{tabular}{|c|c|c|c|c|c|}
\hline \multirow[t]{2}{*}{ Variáveis } & \multicolumn{4}{|c|}{ Níveis de Fósforo $\left(\mathrm{kg} \mathrm{P}_{2} \mathrm{O}_{5} \mathrm{ha}^{-1}\right)$} & \multirow{2}{*}{ Equação de Regressão } \\
\hline & 0 & 40 & 80 & 120 & \\
\hline$\overline{M S V}$ & 1.987 & 2.645 & 3.018 & 2.855 & $\mathrm{Y}=1.973+22,674 \mathrm{X}-0,1267 \mathrm{X}^{2}\left(\mathrm{R}^{2}=0,92\right)$ \\
\hline EUK & - & 16,21 & 12,88 & 7,23 & $Y=21,07-0,1121 X\left(r^{2}=0,89\right)$ \\
\hline $\mathrm{N}$ & 31,76 & 33,41 & 34,12 & 36,02 & $Y=31,81+0,0337 X\left(r^{2}=0,93\right)$ \\
\hline $\mathrm{P}$ & 1,98 & 2,26 & 2,59 & 2,78 & $Y=1,93+0,00682 X\left(r^{2}=0,96\right)$ \\
\hline $\mathrm{Ca}$ & 5,97 & 6,32 & 6,89 & 6,22 & $Y=5,89+0,0224 X-0,00023 X^{2}\left(R^{2}=0,92\right)$ \\
\hline $\mathrm{Mg}$ & 3,27 & 3,98 & 4,19 & 4,02 & $Y=3,21+0,0226 X-0,00012 X^{2}\left(R^{2}=0,94\right)$ \\
\hline $\mathrm{K}$ & 18,54 & 20,98 & 22,33 & 21,15 & $\mathrm{Y}=18,09+0,0908 \mathrm{X}-0,00061 \mathrm{X}^{2}\left(\mathrm{R}^{2}=0,93\right)$ \\
\hline $\mathrm{NN}^{1,2}$ & 11,76 & 19,32 & 25,21 & 27,02 & $Y=13,08+0,1292 X\left(r^{2}=0,93\right)$ \\
\hline $\mathrm{PSN}^{1}$ & 29,55 & 38,17 & 49,51 & 47,78 & $Y=28,71+0,03591 X-0,00162 X^{2}\left(R^{2}=0,90\right)$ \\
\hline
\end{tabular}

${ }^{1}$ Médias de três plantas. ${ }^{2}$ Dados analisados após transformação em $\sqrt{ } \mathrm{x}+1$.

No primeiro caso, a concentração de nutrientes é uma função potencial negativa relacionada ao maior acúmulo de biomassa, notadamente durante o período vegetativo, sendo acentuada com baixa disponibilidade de nutrientes no solo, que não supre adequadamente os requerimentos nutricionais da planta. No segundo caso, quando há competição entre plantas, um dos mecanismos para aumentar a interceptação da radiação incidente é o aumento da estatura da planta, a qual deve investir nutrientes, notadamente o $\mathrm{N}$, para a produção de hastes, estruturas que apresentam baixa concentração de nutrientes (Lemaire et al., 2008). Em pastagens de $S$. guianensis cv. Mineirão, Costa et al. (2006) constataram maiores concentrações de N, P, Ca e Mg com a aplicação de doses entre 60 e $80 \mathrm{~kg}$ de $\mathrm{P}_{2} \mathrm{O}_{5} \mathrm{ha}^{-1}$ conjuntamente com $40 \mathrm{~kg}$ de $\mathrm{K}_{2} \mathrm{O} \mathrm{ha}^{-1}$, enquanto que os teores e as quantidades absorvidas de $\mathrm{P}$ foram diretamente proporcionais às doses aplicadas $\left(0,40,80,120\right.$ e $160 \mathrm{~kg}$ de $\mathrm{P}_{2} \mathrm{O}_{5}$ ha $\left.^{-1}\right)$.

As concentrações de macronutrientes da leguminosa atenderiam, satisfatoriamente, aos requerimentos mínimos dos ruminantes por $\mathrm{N}$, independentemente dos níveis de adubação fosfatada, considerando-se que teores menores que $11,2 \mathrm{~g} \mathrm{~kg}^{-1}$ de MS são limitantes para a adequada fermentação ruminal, implicando em menor consumo voluntário, redução na digestibilidade da forragem e balanço nitrogenado negativo (Minson et al., 1984). Os teores de $\mathrm{Ca}, \mathrm{Mg}, \mathrm{K}$ e $\mathrm{P}$, em todos os níveis de adubação fosfatada foram superiores aos níveis críticos para bovinos de corte em crescimento $\left(1,8 ; 1,0 ; 6,5\right.$ e $1,8 \mathrm{~g} \mathrm{~kg}^{-1}$, respectivamente) sugeridos pelo $\underline{\mathrm{NRC}(2000)}$.

O NCI de P, relacionado com $90 \%$ da produção máxima de $\mathrm{MSV}$, foi estimado em $2,43 \mathrm{~g} \mathrm{~kg}^{-1}$ e obtido com a aplicação de $67,2 \mathrm{~kg}$ de $\mathrm{P}_{2} \mathrm{O}_{5} \mathrm{ha}^{-1}$, sendo inferior aos reportados por Costa et al. (2006) para S. guianensis cv. Mineirão (2,57 $\left.\mathrm{g} \mathrm{kg}^{-1}\right)$ e Costa et al. (2015) para Arachis pintoi cv. Amarillo $\left(2,71 \mathrm{~g} \mathrm{~kg}^{-1}\right)$, evidenciando elevada eficiência de utilização de $\mathrm{P}$ pela leguminosa para produção de forragem, pois o NCI representa a concentração do nutriente abaixo da qual o rendimento é reduzido e acima não apresenta retorno econômico (Nabinger \& Carvalho, 2009).

$\mathrm{O}$ efeito da adubação fosfatada sobre o número de nódulos planta $^{-1}$ foi positivo e linear, enquanto que para o peso seco de nódulos a relação foi quadrática e o máximo valor estimado com a aplicação de $111,9 \mathrm{~kg}$ de $\mathrm{P}_{2} \mathrm{O}_{5}$ ha $^{-1}$ (Tabela 1), evidenciando a existência de efeito compensatório entre número e peso seco de nódulos. Para Acacia angustissima, Costa et al. (2007) reportaram efeito linear da adubação fosfatada $\left(0,40,80\right.$ e $120 \mathrm{~kg}$ de $\mathrm{P}_{2} \mathrm{O}_{5}$ ha $\left.^{-1}\right)$ sobre o número e peso seco de nódulos, contudo para $S$. macrocephala cv. Pioneiro, a máxima nodulação 
foi observada com a aplicação de doses entre $60 \mathrm{e}$ $80 \mathrm{~kg}$ de $\mathrm{P}_{2} \mathrm{O}_{5}$ ha $^{-1}$ (Costa et al., 2006). Para $S$. guianensis cv. Mineirão, Lopes et al. (2011) constataram maior número de nódulos com a aplicação de $400 \mathrm{mg}$ de $\mathrm{P} \mathrm{dm}^{-3}$ de solo, enquanto que para a produção de MS de raízes o máximo valor foi obtido com a aplicação de $257 \mathrm{mg}$ de $\mathrm{P}$ $\mathrm{dm}^{-3}$ de solo.

\section{Conclusões}

A adubação fosfatada afeta positivamente a disponibilidade de forragem, a nodulação e os teores de $\mathrm{N}, \mathrm{P}, \mathrm{Ca}, \mathrm{Mg}$ e $\mathrm{K}$ da leguminosa. A eficiência de utilização de $\mathrm{P}$ é inversamente proporcional às doses aplicadas. A dose de máxima eficiência técnica na produção de MSV foi estimada em $89,4 \mathrm{~kg}$ de $\mathrm{P}_{2} \mathrm{O}_{5}$ ha $^{-1}$ e o nível crítico interno de $\mathrm{P}$, relacionado com $90 \%$ do rendimento máximo de $\mathrm{MSV}$, em 2,43 $\mathrm{g} \mathrm{kg}^{-1}$.

\section{Referências Bibliográficas}

Carneiro, R. F. V., Martins, M. A., Araújo, A. S. F. \& Nunes, L. A. P. L. 2011. Inoculação micorrízica arbuscular e adubação fosfatada no cultivo de forrageiras consorciadas. Archivos de Zootecnia, Belo Horizonte, 60, 1191-1202.

Costa, N., Deschamps, C. \& Moraes, A. 2012. Estrutura da pastagem, fotossíntese e produtividade de gramíneas forrageiras. PUBVET, Londrina, 6, 1387-1392.

Costa, N. L., Gianluppi, V., Braga, R. M., Bendahan, A. B., Mattos, P. S. R., Vilarinho, A. A. \& Oliveira, J. M. F. 2009. Alternativas tecnológicas para a pecuária de Roraima. Boa Vista: Embrapa Roraima. 35p. (Documentos, 19).

Costa, N. L., Magalhães, J. A., Pereira, R. G. A., Townsend, C. R. \& Oliveira, J. R. C. 2007. Considerações sobre o manejo de pastagens na Amazônia Ocidental. Revista do Conselho Federal de Medicina Veterinária, Brasília, 40, 37-56.

Costa, N. L., Paulino, V. T., Magalhães, J. A., Rodrigues, B. H. N. \& Rodrigues, A. N. A. 2015. Adubação fosfatada na produção e composição química da forragem de Arachis pintoi cv. Amarillo. PUBVET, Londrina, 9, 5260.

Costa, N. L., Townsend, C. R., Magalhães, J. A., Paulino, V. T. \& Pereira, R. G. A. 2006.
Formação e manejo de pastagens na Amazônia do Brasil. Revista Electrónica de Veterinária, Madrid, 7, 1-8.

Fabrice, C. E. S., Soares Filho, C. V., Pinto, M. F., Perri, S. H. V., Cecato, U. \& Mateus, G. P. 2015. Recuperação de pastagens de Brachiaria decumbens degradada com introdução de Stylosanthes e adubação fosfatada. Revista Brasileira de Saúde e Produção Animal, Salvador, 16, 758-771.

Gianluppi, V., Smiderle, O. J. \& Gianluppi, D. 2002. Utilização e cultivo de estilosantes lavradeiro nas áreas de cerrado de Roraima. Boa Vista: Embrapa Roraima. 12p. (Circular Técnica, 2).

Lemaire, G. \& Agnusdei, M. 2000. Leaf tissue turnover and efficiency of herbage utilization. In 'Grassland ecophysiology and grazing ecology'. (Eds. G Lemaire, J Hodgson, A de Moraes, PC de F Carvalho, C Nabinger). pp. 265-287: CAB International Publishing: New York.

Lemaire, G., Hodgson, J. \& Chabbi, A. 2011. Grassland productivity and ecosystem services: CABI. 287p.

Lemaire, G., van Oosterom, E., Jeuffroy, M. H., Gastal, F. \& Massignam, A. 2008. Crop species present different qualitative types of response to $\mathrm{N}$ deficiency during their vegetative growth. Field Crops Research, Paris, 105, 253-265.

Lopes, J., Evangelista, A. R., Fortes, C. A., Pinto, J. C., Furtini Neto, A. E. \& Souza, R. M. 2011. Nodulação e produção de raízes do estilosantes Mineirão sob efeito de calagem, silicatagem e doses de fósforo. Ciência $e$ Agrotecnologia, Lavras, 35, 99-107.

Moura, R. L., Nascimento, M. P. S. C. B., Rodrigues, M. M., Oliveira, M. E. \& Lopes, J. B. 2011. Razão folha/haste e composição bromatológica da rebrota de estilosantes Campo Grande em cinco idades de corte. Acta Scientiarum. Animal Sciences, Londrina, 33, 249-254.

Minson, D. J. 1984. Effects of chemical and physical composition of herbage eaten upon intake. In: Hacker, J.B. (Ed.). Nutritional limits to animal production from pasture. Farnham Royal: CAB, p.167-182.

Nabinger, C. \& Carvalho, P. 2009. Ecofisiología de sistemas pastoriles: aplicaciones para su 
sustentabilidad. Agrociencia, Montevideo, 13, 18-27.

NRC. 2000. National Research Council. Nutrient requirements of beef cattle. $7^{\text {th }}$ ed. Washington: National Academy Press, 242p.

Otsubo, A. A., Brito, O. R., Schnitzer, J. A. \& Otsubo, V. H. N. 2011. Produção de matéria seca de Stylosanthes capitata submetido à adubação fosfatada em solos com diferentes texturas Semina: Ciências Agrárias, Londrina, 32, 1677-1686.

Silva, D. \& Queiroz, A. C. 1981. Análise de alimentos:métodos químicos e biológicos. Viçosa: UFV, Impr. Univ. 235p.

Silva, F. C. 2009. Manual de análises químicas de solos, plantas e fertilizantes. Embrapa
Informação Tecnológica. Brasília, DF. 370p.

Simão Neto, M., Gonçalves, C. A., Camarão, A. P. \& Dutra, S. 1999. Adubação e calagem de leguminosas tropicais (Pueraria phaseoloides e Stylosanthes guianensis cv. Cook) no Nordeste paraense, Brasil. Pasturas Tropicales, Cali, 21, 18-24.

Article History:

Received 11 March 2018

Accepted 31 March 2018

Available online 4 May 2018

License information: This is an open-access article distributed under the terms of the Creative Commons Attribution License 4.0, which permits unrestricted use, distribution, and reproduction in any medium, provided the original work is properly cited. 